\title{
technologies
}

ISSN 2227-7080

www.mdpi.com/journal/technologies

Article

\section{Wireless Accelerometer for MRI-Guided Interventional Procedures}

\author{
Martyn N.J. Paley ${ }^{1}{ }^{*}$, Araminta Ledger ${ }^{2}$, Martin O. Leach ${ }^{2}$, Craig Cummings ${ }^{2}$, \\ Raymond Hughes ${ }^{3}$ and Ali Akgun ${ }^{3}$
}

1 Unit of Academic Radiology, University of Sheffield, Sheffield S10 2JF, UK

2 CR-UK and EPSRC Cancer Imaging Centre, Institute of Cancer Research, Royal Marsden NHS Foundation Trust, 15 Cotswold Road, Belmont, Sutton, Surrey SM2 5NG, UK;

E-Mails: minty.ledger@icr.ac.uk (A.L.); martin.leach@icr.ac.uk (M.O.L.); craig.cummings@icr.ac.uk (C.C.)

3 Specialty Magnetics Limited, Unit-1, 10 Stonefield Way, South Ruislip, Middlesex HA4 0JS, UK; E-Mails: r.hughes@ specialtymagnetics.com (R.H.); aliakgun@specialtymagnetics.com (A.A.)

* Author to whom correspondence should be addressed; E-Mail: m.n.paley@ shef.ac.uk; Tel.: +44-114-271-3208; Fax: +44-114-272-4760.

Received: 6 October 2013; in revised form: 26 November 2013 / Accepted: 2 December 2013 / Published: 10 December 2013

\begin{abstract}
MRI-guidance is increasingly used for minimally-invasive procedures, such as biopsy, and requires real-time active tracking of surgical instruments. Although optical and MR-based fiducial tracking devices have been used, these systems rely on complex contact with the operator or line-of-sight access for effective operation. A more straight-forward and clinically robust method is required to allow interactive real-time slice positioning of MR scan planes during interventional procedures. This study evaluated the use of a wristwatch-mounted, low cost wireless interface device for real-time MRI guidance. The device was designed to interact with software for planning rather than instrument guidance. The wireless device was integrated with two novel, open interventional magnet systems operating at $0.17 \mathrm{~T}$ and $0.5 \mathrm{~T}$ and utilized a novel customized graphic user interface (GUI) to assess interventional capability.
\end{abstract}

Keywords: interventional MRI; wireless accelerometer; geometry planning; probe navigation 


\section{Introduction}

Magnetic resonance imaging (MRI) guidance of interventional procedures provides a number of advantages: it avoids the radiation exposure of computed tomography (CT) and improves upon the low soft tissue contrast of ultrasound. In particular, MRI guidance has been adopted for minimally-invasive procedures such as biopsy and tissue ablation in breast, prostate, orthopaedic and vascular applications [1-5]. A number of specialist interventional MR scanners, often with open configuration, have been employed effectively for minimally-invasive procedures [6,7].

Real time interactive slice positioning of scan planes is required to allow tracking of biopsy needles or surgical instruments during interventional MR procedures. However, the presence of static and time-varying magnetic fields creates a difficult environment for device localization, in terms of both system operation and safety considerations. Various interface devices such as trackballs and joysticks as well as optical and MR-based fiducial tracking devices have been used previously but usually need complex contact with the operator or line of sight access for correct operation [5,6]. Widespread clinical adoption of MRI-guidance for clinical procedures will require more straight-forward visualization of instrument and target tissue within the field-of-view (FOV). Furthermore, this needs to be coupled with a robust interactive graphical user interface (GUI). This study investigated the use of a low cost wireless interface device to track controlled rotational motions produced by the operator in order to adjust the MR scan plane in real time. For ease of use, the system was linked to a customised GUI which allowed the operator to control the device from an adjacent MR-compatible touchscreen. The wireless device was integrated with two novel interventional magnet systems $(0.17 \mathrm{~T}$ and $0.5 \mathrm{~T})$ to evaluate operational capability.

\section{Experimental Section}

A wireless accelerometer, conveniently housed in a waterproof watch casing which could be worn by an interventional surgeon below surgical gloves and operating at $868 \mathrm{MHz}$ (EZ-Chronos, Texas Instruments, Austin, TX, USA) (Figure 1), was interfaced to the lesion localization and probe targeting software of the specialized MRI systems written in LabView (National Instruments, Austin, TX, USA). The acceleration outputs of the device were allocated to two plane rotation angles and to the slice offset or field of view (FOV) offset as chosen by the operator. Zero position was set with the watch located horizontally and pointing into the magnet. Rotation of the accelerometer by \pm 90 degrees around two axes was then calibrated and used to adjust the selected scan plane angle and slice offset interactively. The accelerometer was zeroed in the horizontal plane and calibrated so that a 90 degree rotation in physical space achieved by moving the forearm corresponded to a \pm 90 degree plane rotation or a $\pm F O V / 2$ slice offset depending on the direction of rotation. Worn by the operator, the accelerometer device would allow the surgeon to use his wrist to interact directly with the software to plan the next scan plane. The device is not specifically designed to track the location of an instrument or biopsy device but to give the surgeon a non-contact method of interacting with planning software. 
Figure 1. (a) The wireless accelerometer (EZ Chronos model 430, Texas Instruments, Austin, Tx, USA) is housed in a sports watch and transmits data from the accelerometer over a $868 \mathrm{MHz}$ radio link to a USB wireless transceiver. Labview software is used to receive the wireless data and interface it to the lesion localization and probe targeting software of specialized interventional MRI systems with a $50 \mathrm{~ms}$ update time. (b) The customized Labview graphical user interface (GUI). (c) The precise geometry of the inserts in the quality assurance (QA) phantom used to assess the angular $\left( \pm 3^{\circ}\right)$ and translational ( $\pm 1 \mathrm{~mm}$ ) accuracy of the geometrical planning through the wireless interface. The phantom was $90 \mathrm{~mm}$ internal length. Angles and offsets of acquired slices were calculated using the visible spatial features and dimensions of the QA phantom in each planned slice using standard geometrical methods and ImageJ analysis software (NIH, Bethesda, MD, USA).

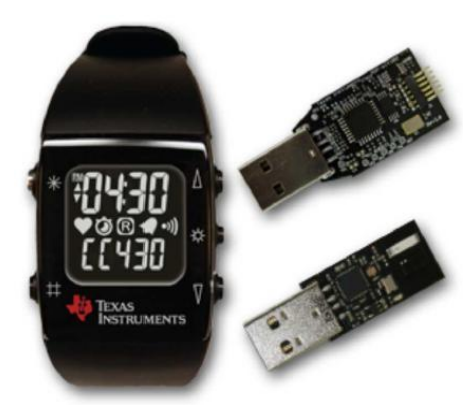

(a)

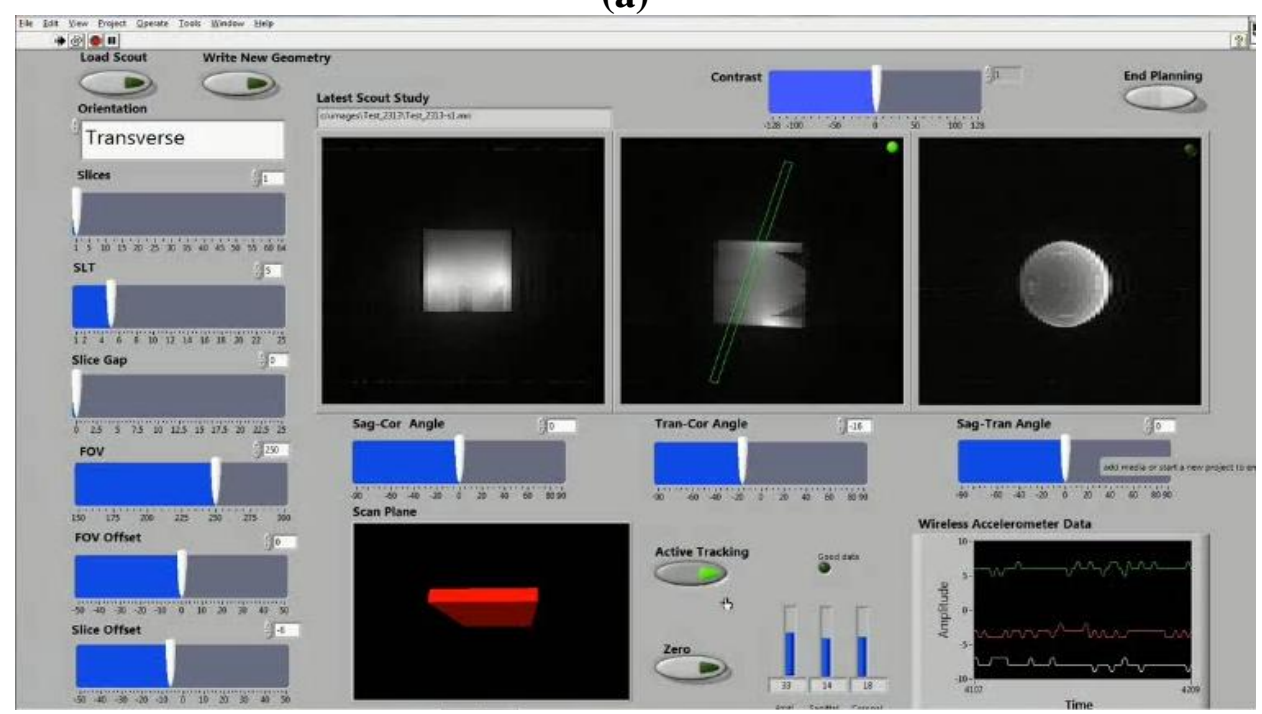

(b)
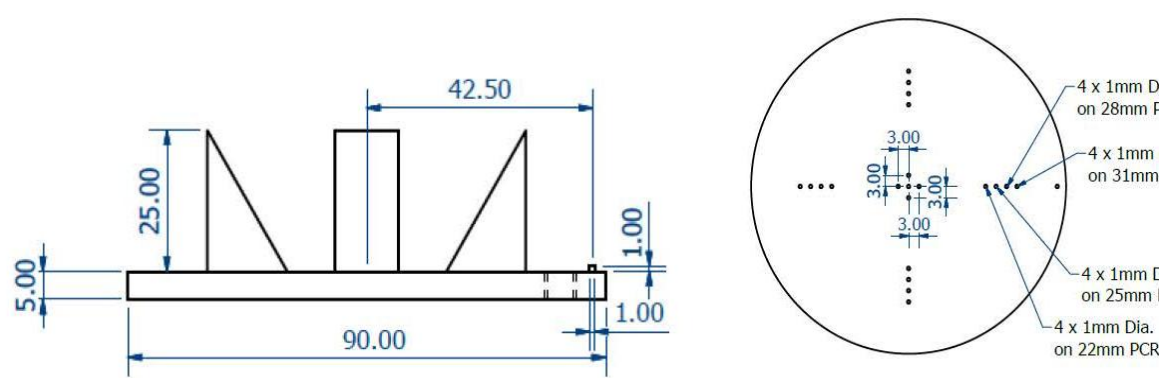

(c) 
Testing was performed on a dedicated 0.17T MR orthopaedic and neonatal scanner (InnerVision MRI Ltd., Bradley, UK) (Figure 2a) and also an Intra-Operative MR Scanner (Specialty Magnetics Limited, Middlesex, UK) (Figure 3) currently operated at $0.5 \mathrm{~T}$ for occupational health reasons and optimised for minimally invasive breast procedures. Both systems used LabView based research spectrometers with custom-designed interactive GUIs (ISD Ltd., Bradley, UK). A USB PC interface to the wireless accelerometer, including a low power RF transceiver (TI CC1111, Texas Instruments, Austin, TX, USA), was housed in the scan computer located outside the screened room of each system to receive the wireless data stream.

Angular accuracy and slice offset were assessed at $0.17 \mathrm{~T}$ using a customized quality assurance (QA) phantom (90 mm height $\times 90 \mathrm{~mm}$ OD) (Figure 1c). Wireless planned oblique images of the QA phantom with defined geometry and the elbow region of a volunteer were acquired at $0.5 \mathrm{~T}$ and $0.17 \mathrm{~T}$ respectively, with a T1-weighted spin echo sequence $(\mathrm{TR} / \mathrm{TE}=300 / 20 \mathrm{~ms})$ with $1 \mathrm{~mm}$ in-plane resolution, $\mathrm{SLT}=5 \mathrm{~mm}, \mathrm{NEX}=2$ (Figures $1 \mathrm{~b}$ and $2 \mathrm{~b}$, respectively) to assess the oblique planning capability. Through the precisely known geometry of the phantom (Figure 1c), including the slice thickness evaluation ramps and spatial resolution bars, angles could be determined by visualising specific landmarks in the acquired MR images. Images of the QA phantom were also acquired using the T1-weighted spin echo sequence with and without the device present and active within the magnet room to assess for radiofrequency line artifacts or degradation of signal to noise ratio by the wireless device.

Figure 2. (a) A specialised, open low field (0.17T) MRI system used for orthopaedic and neonatal applications providing easy access for interventional procedures (InnerVision MRI Ltd., Bradley, UK). The footprint of the magnet is $500 \times 500 \mathrm{~mm}$ and the iso-centre of the magnet is only $250 \mathrm{~mm}$ from the front face of the magnet covers. (b) Wireless planned oblique image of the elbow region of a volunteer acquired at $0.17 \mathrm{~T}$ with a T1-weighted sequence $(\mathrm{TR} / \mathrm{TE}=300 / 20 \mathrm{~ms})$ with $1 \mathrm{~mm}$ in-plane resolution, $\mathrm{SLT}=5 \mathrm{~mm}$, $\mathrm{NEX}=2$. This image was acquired with the wireless device active at the edge of the uniform magnet volume within the screened room and showed no degradation of signal to noise ratio or line artifacts.

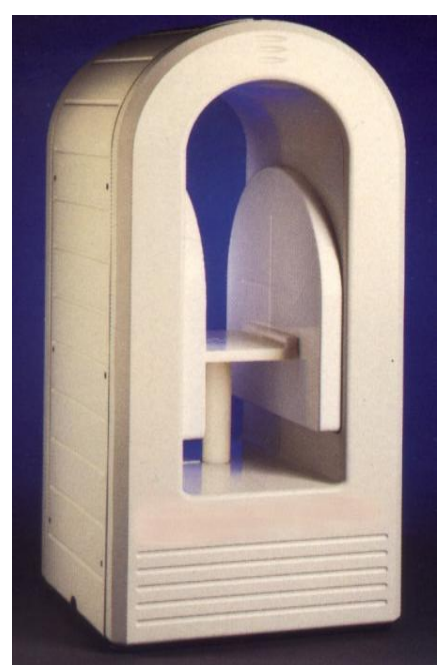

(a)

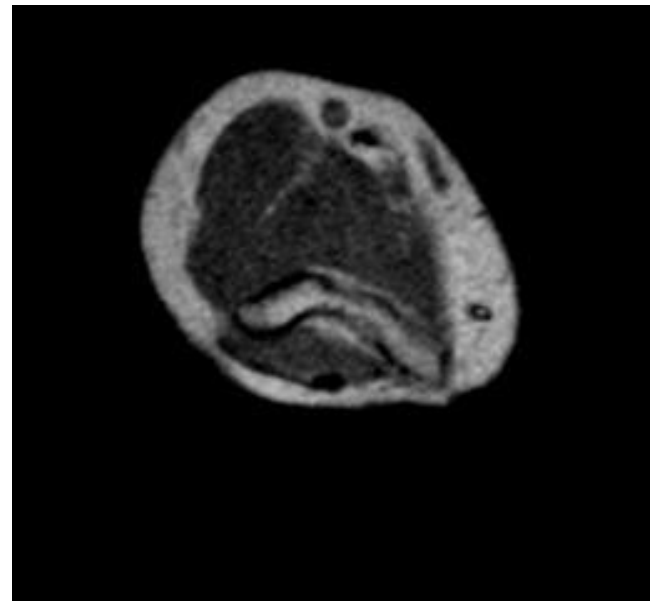

(b) 
Figure 3. (a) A superconducting magnet based Intra-Operative Breast MR Scanner (Specialty Magnetics Limited, Middlesex, UK) currently operated at $0.5 \mathrm{~T}$ for occupational health reasons with $70 \mathrm{~cm}$ bore-length and $70 \mathrm{~cm}$ bore-diameter. (b) MR Scanner features an MR-compatible, in-room, touchscreen Operator's Terminal through which the Clinician(s) can control all the subsystems of the MR Scanner and perform interventional procedures under real-time MRI guidance. MR Scanner also features a highly maneuverable Patient Positioning System which, as well as traditional vertical and horizontal travel, can also rotate clockwise or counter clockwise to locate the breast precisely at iso-centre of the magnet.

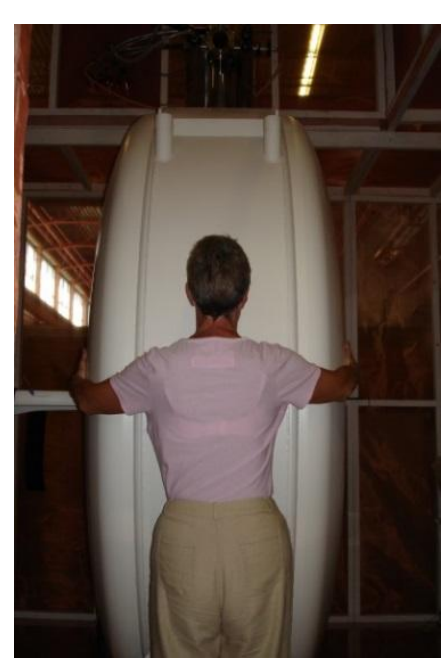

(a)

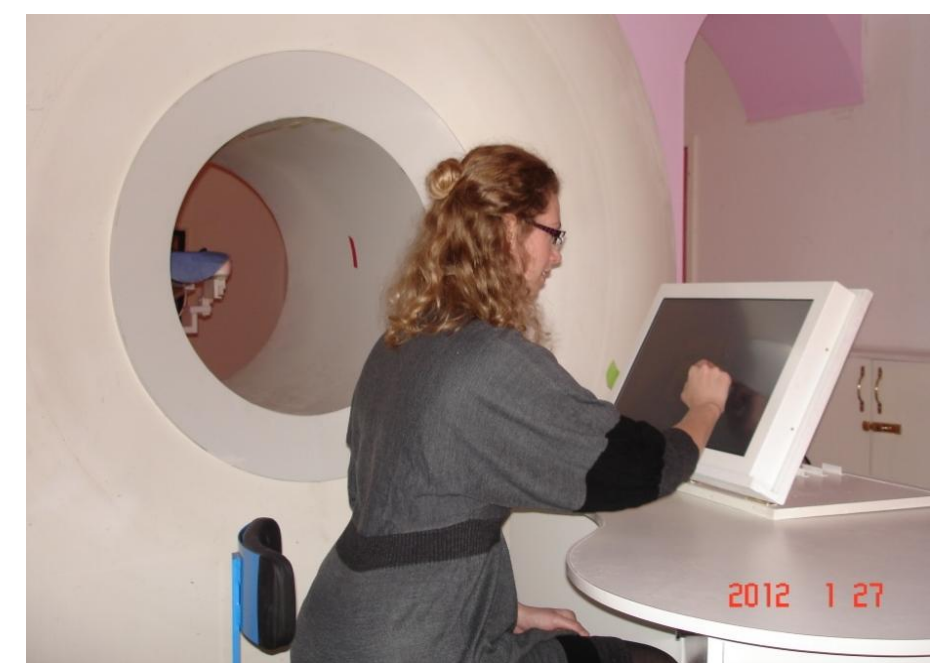

(b)

Figure 4. A simplified version of the LabView wireless interactive geometry planning software showing three scout images acquired at $0.5 \mathrm{~T}$ from the breast phantom in axial, sagittal and coronal planes with the planned slice superimposed in green for the operator.

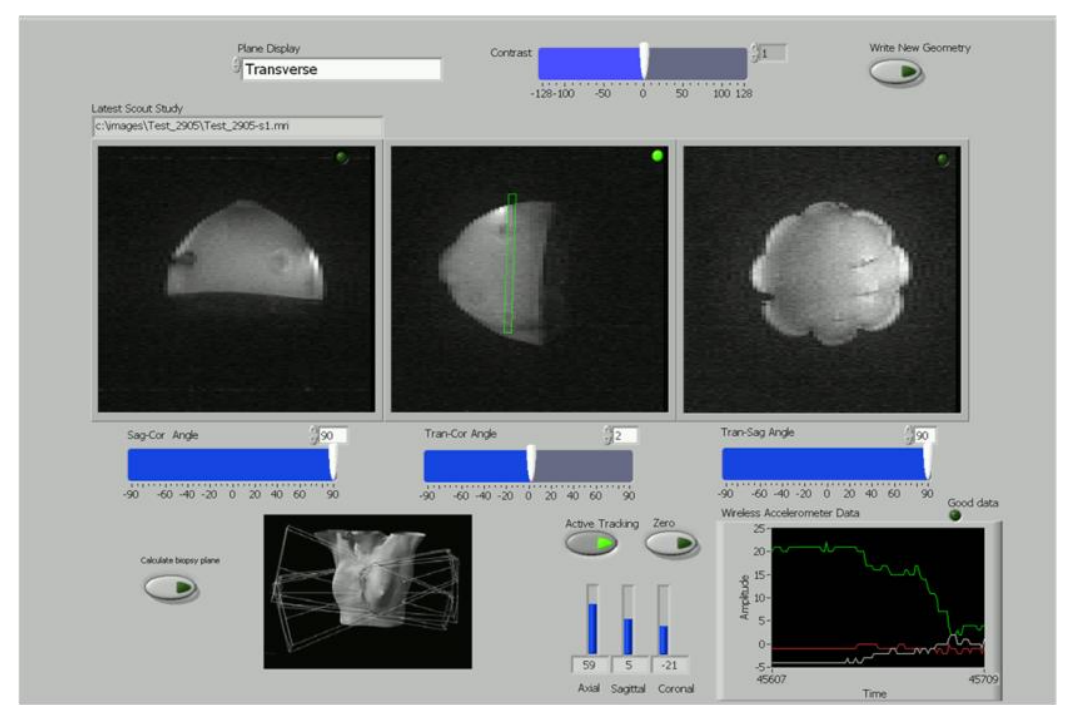

The suitability of the wireless device for MR scan plane adjustment in real time during biopsy was tested using the Intra-Operative Breast MR Scanner (Specialty Magnetics Limited, Middlesex, UK). A touchscreen-compatible GUI for the LabView (National Instruments, Austin, TX, USA) spectrometer 
was constructed (Figure 4). This allowed both the wireless device and MR sequences to be initiated from the face of the MR scanner.

A gelatin-based breast biopsy phantom was employed, containing several spherical inclusions (8-12 mm ID) at varying depths and heights to mimic cysts (pure distilled water), fat (corn oil) and contrast-enhanced tumours (short T1 Gadolinium solutions). These inclusions were used to evaluate lesion targeting with the biopsy probe. A commercially available vacuum-assisted breast biopsy (VABB) system (MR Mammotome System, Devicor Medical Products, Cincinnati, OH, USA) was used to test the biopsy procedure with an $8 \mathrm{G}$ MR-compatible biopsy targeting set (Mammotome MR Targeting Set, $115 \mathrm{~mm}$ length). To avoid susceptibility artifacts during the evaluation, the ceramic obdurator within the biopsy set was used to target inclusions within the breast phantom. The biopsy probe was thus visible within the MR images as a signal void.

A freehand biopsy procedure was performed in two parts: (i) calculation of an initial visualization scan plane containing both needle entry point and lesion; (ii) interactive lesion targeting using the wireless device.

(i) A fiducial marker (short T1 Gadolinium solution) was placed against the phantom in order to locate a nominal needle entry point. A set of low-resolution coronal images (FLASH sequence: $\mathrm{TR} / \mathrm{TE}=60 / 5 \mathrm{~ms}$, matrix $=128 \times 256, \mathrm{SLT}=3 \mathrm{~mm}, \mathrm{NEX}=2$ ) were then acquired to include both the fiducial and the target inclusion. Using the customized GUI, the operator located both objects within the coronal slices and used the software to automatically calculate an initial axial-coronal scan plane containing both objects. This provided an initial visualization scan plane for a free-hand biopsy. By superimposing this initial scan plane on three scout images (axial, sagittal and coronal), the operator could easily visualize the orientation of this plane within the phantom (see planned slice in Figure 4).

(ii) Dynamic images were acquired using a FLASH sequence (TR/TE $=60 / 5 \mathrm{~ms}$, matrix $=64 \times 256$, SLT $=3 \mathrm{~mm}, \mathrm{NEX}=1$ ) with a temporal resolution, including 8 channel 2DFT reconstruction and image display, of $4 \mathrm{~s}$ allowing the biopsy needle to be tracked using a series of 7-8 images during a needle insertion. Starting from the initial visualization scan plane planned in (i), the operator was able to use the wrist-mounted wireless device to interactively adjust this initial imaging scan plane as the probe moved towards the lesion. The linked touchscreen GUI displayed the scan planes selected by the wireless accelerometer during the biopsy procedure together with the targeting images corresponding to the selected plane (Figure 5). The time taken to move the probe to the planned target was typically $30 \mathrm{~s}$.

Figure 5. The track of a biopsy needle acquired at $0.5 \mathrm{~T}$ which has been planned using the wireless planning software.

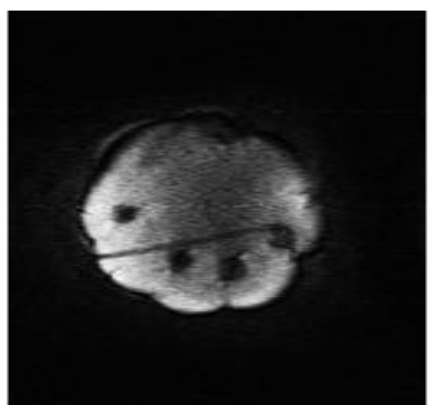




\section{Results and Discussion}

The wireless interface operated effectively with both of the interventional MR scanners (Figures 2a and 3) during image acquisition. The wireless device was not affected by the magnetic or radiofrequency fields during imaging (the device was kept outside the transmit coils) and did not produce interference on the images. This was to be expected given the widely different operating frequencies between the device (868 MHz) and the MRI Larmor frequencies (21.3 MHz and 7.2 MHz at $0.5 \mathrm{~T}$ and $0.17 \mathrm{~T}$, respectively). The USB interface was extended partially into the RF screened room waveguides of each system to provide improved reception with the RF screened room door fully closed.

Figure $1 \mathrm{~b}$ shows a planned slice on the customized QA phantom (green rectangle on central image). Two of the orthogonal accelerometer outputs (shown bottom right) are assigned to slice offset and plane rotation angle respectively. Angular accuracy of \pm 3 degrees and a slice offset of $\pm 1 \mathrm{~mm}$ were found at $0.17 \mathrm{~T}$ using the customized QA phantom. Images of the QA phantom and a volunteer's elbow were acquired at $0.5 \mathrm{~T}$ and $0.17 \mathrm{~T}$ respectively, dependent on the wireless accelerometer output. Typical examples are shown in Figures $1 b$ and $2 b$, respectively.

The wireless device could be used to interactively adjust the scan plane going through both the target-lesion and the biopsy-probe in real time (50 ms update time) based on operator wrist rotation with no other physical contact to the system. During minimally-invasive procedures, this would ensure sterile operation. An easy-to-use graphic user interface (GUI) is essential for greater clinical adoption of interventional MR procedures. Figure 4 displays the simplified version of the LabView wireless interactive geometry planning software which was designed specifically as a touchscreen-compatible GUI for this study. Together with the wireless accelerometer output, large slide-bars were incorporated to give the operator easy control of the imaging sequences. The use of a plastic stylus on the touchscreen GUI gave the user straight-forward simultaneous control of the wireless device and MR imaging whilst maintaining a sterile environment. Although a number of MR systems have included novel GUIs, the majority of these systems still locate the software navigation within the external MRI control room [6,8]. Although projection techniques have been used to good effect, this still isolates the operator from essential real-time control of the procedure $[9,10]$. The touchscreen Operator's Terminal control afforded by the Intra-Operative MR Scanner (Specialty Magnetics Limited, Middlesex, UK) design was highly conducive to effective clinical use of the wireless accelerometer since it allowed tableside sequence triggering and procedure monitoring (Figure 3b). A dynamic imaging sequence enabled the operator to easily target an inclusion using wrist motion to interactively select the image scan plane in real time. Figure 5 displays a typical image acquired mid-way through a biopsy procedure using the breast phantom with both the biopsy probe and target inclusions visible as signal drop out.

Instrument tracking in MRI can be broadly divided into two categories: passive and active. Within the first category, visualization of the probe is deduced from the susceptibility artifact induced by the instrument itself, and guidance is achieved by navigation in relation to passive fiducial markers. Without automatic slice selection of scan planes, passive techniques require manual slice calculation and repositioning which add time to interventional procedures. However, this simpler approach has been widely adopted in clinical settings, in particular for MRI-guided breast biopsy [1,11-13]. In contrast, active tracking gives the operator direct instrument control with immediate feedback under real-time MR image guidance. A number of active tracking techniques have been developed, including 
optical tracking with an infra-red camera and mirrors [14,15] and also the use of MR micro-receiver coils within the device to induce local inhomogeneities within the magnetic field [16-18]. Although optical tracking provides a wireless approach, it requires line-of-sight access for accurate guidance which can be problematic in many MR scanner designs. Localization via micro-receiver coils can also provide a wireless solution if the coils are well tuned, matched and de-coupled to one another but this can be technically challenging and very costly. Consequently, it is more common for micro-coil tracking to be used with a conductive wire which introduces safety issues such as radiofrequency heating [5]. In addition, inhomogeneities within the $\mathrm{B}_{0}$ field can affect micro-coil localization. In contrast, the wireless accelerometer trialled within this study was able to provide an effective low-cost, wireless solution that did not interfere with MR imaging and conferred the added advantage of sterile operation.

This study was subject to a number of limitations. The wireless accelerometer has been shown to provide real-time plane rotation and slice offset information to the operator. However, at the time of this study, the MR sequences available to us were too slow to provide real-time image iteration during the phantom biopsy procedure. However, the use of FLASH was able to demonstrate proof of principle and subsequent work will test the accelerometer with more rapid sequences. In addition, although the wireless accelerometer device within this study was evaluated on interventional MR systems operated at relatively low magnetic field systems, translation of the device to much higher-field MR systems would also be feasible.

This study presents the first attempt to use a wireless accelerometer for active instrument tracking within the MR environment. It establishes that a watch-mounted accelerometer can provide sterile, cost-effective and straight-forward user control for interventional procedures under MR guidance.

\section{Conclusions}

Interactive scan plane control has been demonstrated using a wireless accelerometer device which allows rapid and precise control of the slice angle and offset, from simple rotational movements of the accelerometer device, providing sterile and position independent interaction with the MR geometry planning software.

\section{Acknowledgments}

(1) We acknowledge the support received from UK's Technology Strategy Board in the form of a grant awarded to Specialty Magnetics Limited as well as the additional support received from Specialty Magnetics Limited in the form of a "matching fund".

(2) We also acknowledge the support received for the CR-UK and EPSRC Cancer Imaging Centre in association with the MRC and Department of Health (England) (grants C1060/A10334 and C16412/A6269) NHS funding to the NIHR Biomedical Research Centre.

\section{Conflicts of Interest}

Araminta Ledger (The Institute of Cancer Research, UK) has been funded by Specialty Magnetics Limited through the grant award received from the UK's Technology Strategy Board. Martin O. Leach 
holds a non-remunerated directorship of Specialty Scanners plc (Middlesex, UK), parent company of Specialty Magnetics Limited. Martin O Leach is an NIHR Senior Investigator. Martyn N.J. Paley is a director of Imaging Systems Design Ltd. and InnerVision MRI Ltd.

\section{References}

1. Heywang-Köbrunner, S.H.; Heinig, A.; Pickuth, D.; Alberich, T.; Spielmann, R.P. Interventional MRI of the breast: Lesion localisation and biopsy. Eur. Radiol. 2000, 10, 36-45.

2. Postma, E.L.; van Hillegersberg, R.; Daniel, B.L.; Merckel, L.G.; Verkooijen, H.M.; van den Bosch, M.A.A.J. MRI-guided ablation of breast cancer: Where do we stand today? J. Magn. Reson. Imaging 2011, 34, 254-261.

3. Morrison, P.R.; Silverman, S.G.; Tuncali, K.; Tatli, S. MRI-guided cryotherapy. J. Magn. Reson. Imaging 2008, 420, 410-420.

4. Seebauer, C.J.; Hermann, J.B.; Rump, J.C.; Walter, T.; Teichgräber, U.K.M. Advancements in orthopedic intervention: Retrograde drilling and bone grafting of osteochondral lesions of the knee using magnetic resonance imaging guidance. Cardiovasc. Intervent. Radiol. 2010, 33, 1230-1234.

5. Kos, S.; Huegli, R.; Bongartz, G.M.; Jacob, A.L.; Bilecen, D. MR-guided endovascular interventions: A comprehensive review on techniques and applications. Eur. Radiol. 2008, 18, 645-657.

6. Blanco, R.T.; Ojala, R.; Kariniemi, J.; Perälä, J.; Niinimäki, J.; Tervonen, O. Interventional and intraoperative MRI at low field scanner-A review. Eur. J. Radiol. 2005, 56, 130-142.

7. Pondman, K.M.; Fu, J.J.; Schultze, L.J.; Witjes, J.A.; Hambrock, T.; Macura, K.J.; Barentsz, J.O. MR-guided biopsy of the prostate: An overview of techniques and a systematic review. Eur. Urol. 2008, 54, 517-527.

8. Jyrkinen, L.; Ojala, R.; Haataja, L.; Blanco, R.; Klemola, R.; Silven, O.; Tervonen, O. Managing the Complexity of the User Interface of a MRI Guided Surgery System. In Proceedings of 6th ERCIM Workshop "User Interfaces for All”, University of Oulu, Florence, Italy, 25-26 October 2000; pp. 213-219.

9. Wildermuth, S.; Debatin, J.F.; Leung, D.A.; Dumoulin, C.L.; Darrow, R.D.; Uhlschmid, G.; Hofmann, E.; Thyregod, J.; von Schulthess, G.K. MR-imaging guided intravascular procedures: Initial demonstration in a pig model. Radiology 1997, 202, 578-583.

10. Beyersdorff, D.; Winkel, A.; Hamm, B.; Lenk, S.; Loening, S.A.; Taupitz, M. MR imaging-guided prostate biopsy with a closed MR unit at $1.5 \mathrm{~T}$ : Initial results. Radiology 2005, 234, 576-581.

11. Ojeda-Fournier, H.; Mahoney, M.C. Part 1: MR-guided breast interventions: Pearls and pitfalls. Appl. Radiol. 2011, 40, 18-25

12. Ojeda-Fournier, H.; Mahoney, M.C. Part 2: MR-guided breast interventions: Pearls and pitfalls. Appl. Radiol. 2011, 40, 6-22.

13. Mahoney, M.C.; Newell, M.S. Breast intervention: How I do it. Radiology 2013, 268, $12-24$.

14. Ojala, R.; Klemola, R.; Karppinen, J.; Sequeiros, R.B.; Tervonen, O. Sacro-iliac joint arthography in low back pain: Feasibility of MRI guidance. Eur. J. Radiol. 2001, 40, 236-239. 
15. Sequeiros, R.B.; Klemola, R.; Ojala, R.; Jyrkinen, L.; Lappi-Blanco, E.; Soini, Y.; Tervonen, O. MRI-guided trephine biopsy and fine-needle aspiration in the diagnosis of bone lesions in low-field (0.23 T) MRI system using optical tracking. Eur. Radiol. 2002, 12, 830-835.

16. Joensuu, R.P.; Sepponen, R.E.; Lamminen, A.E.; Standertskjöld-Nordenstam, G.G.M. A shielded Overhauser marker for MR tracking of interventional devices. Magn. Reson. Med. 2000, 43, 139-145.

17. Flask, C.; Elgort, D.; Wong, E.; Shankaranarayanan, A.; Lewin, J.; Wendt, M.; Duerk, J.L. A method for fast 3D tracking using tuned fiducial markers and a limited projection reconstruction FISP (LPR-FISP) sequence. J. Magn. Reson. Imaging 2001, 14, 617-627.

18. Susil, R.C.; Yeung, C.J.; Atalar, E. Intravascular extended sensitivity (IVES) MRI antennas. Magn. Reson. Med. 2003, 50, 383-390.

(C) 2013 by the authors; licensee MDPI, Basel, Switzerland. This article is an open access article distributed under the terms and conditions of the Creative Commons Attribution license (http://creativecommons.org/licenses/by/3.0/). 